\title{
Rursus
}

Russus

Poiétique, réception et réécriture des textes antiques

9 | 2016

Commentaires anciens (pragmatique \& rhétorique)

\section{La catabase entre parenthèses : Tiberius Claudius Donat et le chant 6 de l'Énéide}

Putting the catabasis in brackets: Tiberius Claudius Donatus on Aeneid 6

Séverine Clément-Tarantino

\section{(2) OpenEdition}

Journals

Édition électronique

URL : http://journals.openedition.org/rursus/1212

DOI : $10.4000 /$ rursus. 1212

ISSN : 1951-669X

Éditeur

Université Nice-Sophia Antipolis

Référence électronique

Séverine Clément-Tarantino, "La catabase entre parenthèses : Tiberius Claudius Donat et le chant 6 de l'Énéide », Rursus [En ligne], 9 | 2016, mis en ligne le 29 juillet 2016, consulté le 19 avril 2019. URL: http://journals.openedition.org/rursus/1212; DOI : 10.4000/rursus.1212

Ce document a été généré automatiquement le 19 avril 2019

Rursus 


\title{
La catabase entre parenthèses : Tiberius Claudius Donat et le chant 6 de l'Énéide
}

\author{
Putting the catabasis in brackets: Tiberius Claudius Donatus on Aeneid 6
}

Séverine Clément-Tarantino

1 L'idée que le chant 6 de l'Énéide constitue le cœur de l'épopée de Virgile est difficilement contestable: il en est le cœur non seulement par sa place, mais également par l'expérience hors du commun qu'il fait vivre à son héros, la richesse, la densité, la complexité de ses significations ${ }^{1}$. Cette complexité, cependant, tient beaucoup au fait que c'est aussi le chant le plus mythologique du poème - tout en intégrant la leçon des plus éloquents critiques du mythe, notamment Lucrèce $^{2}$, Virgile procède à une remythologisation des enfers. Mais il ne s'agit pas non plus de restaurer, pour ellesmêmes, les histoires terrifiantes, en particulier, de grands criminels et châtiments; celles-ci se voient explicitement attacher une finalité et une signification morales ${ }^{3}$. Par ailleurs, certaines données mythologiques sont l'objet d'une mise à distance, laquelle peut fonctionner comme une invitation à la lecture allégorique ${ }^{4}$. Bien plus, quand Virgile clôt la catabase en faisant sortir Énée par la porte d'ivoire, celle des falsa... insomnia, il peut sembler signifier que tout "ce qu'il a dit a été inventé »". Dans l'Énéide de Paul Veyne ${ }^{6}$, cela veut dire que le chant 6 a encore plus la saveur d'un conte, au sein d'une Énéide allègre et vive, et qui n'a rien de mystique, ni même de religieux; pour un Servius et d'autres lecteurs païens et chrétiens de Virgile, c'est un encouragement de plus à chercher et à développer le sens à un autre, et même à d'autres niveaux?

2 Tiberius Claudius Donat (TCD), commentateur dont l'activité n'a sans doute pas été très éloignée dans le temps de celle de Servius ${ }^{8}$, ne s'intéresse pas aux profondeurs du poème virgilien telles qu'elles sont explorées, notamment, par Servius et Macrobe ${ }^{9}$. L'enjeu de son commentaire, les Interpretationes Vergilianae (désormais I. V.) n'est pas de dévoiler un sens enfoui et crypté par Virgile dans ses vers, mais plutôt de déployer et de faire rayonner la richesse des significations que le poète a encloses dans des mots remarquables de brièveté et de densité ${ }^{10}$. Le Virgile de Donat est un maître de vie et le 
plus grand rhéteur ${ }^{11}$; quoiqu'il soit un peritissimus doctor en tous domaines ${ }^{12}$, il n'est ni religieux ni philosophe ${ }^{13}$. Mais alors, en considérant en outre que ce commentateur se soucie peu des intertextes et n'étudie pas systématiquement les mythes, on peut se demander quel intérêt le chant 6 peut encore comporter pour lui.

\section{La catabase, une digression entre deux épitaphes}

3 La question se pose de manière encore plus pressante si, avant même de considérer de près la lecture que TCD fait de l'épisode de la descente aux enfers, on tombe sur le commentaire qu'il donne des premiers mots du chant 7 de l'Énéide, le début de l'épitaphe en l'honneur de la nourrice d'Énée, Caieta (Aen. 7, 1-2):

Tu quoque litoribus nostris, Aeneia nutrix, aeternam moriens famam, Caieta, dedisti,

Toi aussi, nourrice d'Énée, tu as donné, en mourant, une renommée éternelle à nos rivages.

4 C'est sur l'adverbe quoque que bute et se concentre le commentateur ${ }^{14}$. Il évoque (ou soulève lui-même) en effet une quaestio, disant que (I. V. II, p. 3, 1. 18-20)

peut-être quelqu'un pourrait penser que le début que Virgile a imaginé pour ce livre n'est pas approprié parce qu'il a dit 'toi aussi' (tu quoque), alors que personne ne semble avoir été nommé avant ce vers.

Habituellement, dans l'esprit du lecteur moderne, le lien se fait immédiatement avec les autres "épitaphes" dédiées à des compagnons d'Énée que sont les v. 870-871 du chant 5 et les v. 232-235 du chant 6 respectivement dédiées à Palinure (avec une apostrophe au personnage - c'est Énée qui parle) et Misène (on est alors dans le discours du narrateur). Certes, quoque peut être d'abord source de surprise dans l'incipit du chant 7, mais surprise et gêne éventuelle sont vite dissipées si l'on enchaîne la lecture des chants 6 et 7 : l'apostrophe à Caieta et le contenu des v. 1-4 éclairent la mention proleptique de Gaète au v. 900 du chant 6, en même temps qu'elle consacre le procédé étiologique antérieurement mis en œuvre à propos de Misène et Palinure. Pour résoudre le problème soulevé, TCD ne raisonne pas différemment; simplement, il paraît le faire d'une manière bien plus littérale et drastique. Quoque n'est problématique que pour qui n'est pas capable de « retrancher ce qui a été jeté dans l'entre-deux » (qui interiecta nesciunt retrahere, I.V. II, p. $3,1.20-p .4,1.1)$ - entre l'évocation du tombeau de Misène et l'évocation de la mort et du tombeau de Caieta. Or, ce qui se trouve dans cet entre-deux n'est autre que le récit de la descente d'Énée dans le monde d'en-bas ${ }^{15}$, le « développement sur les enfers » (I.V. II, p. 4, 1. 1-17) $)^{16}$ :

Iste enim uersus sublato medio de inferis tractatu Miseni exequiis iungitur ; ab his enim discedens carminis cursus ad easdem redit et facit integram narrationem, ut recte posuerit 'tu quoque'. Melius autem apparebit haec adsertio si unde discessum est repetamus : 'at pius Aeneas ingenti mole sepulchrum imponit suaque arma uiro remumque tubamque monte sub Aerio qui nunc Misenus ab illo dicitur aeternumque tenet per saecula nomen.' Sequi debuit 'tu quoque litoribus nostris, Aeneia nutrix, aeternam moriens famam, Caieta, dedisti'. Interposita igitur de inferis carmina quod unum esse oportuit diuiserunt. Sed et nunc unum est, quia unde se recessisse meminerat poeta remeauit. Ergo recte superioribus haec nectens ait 'tu quoque litoribus nostris, Aeneia nutrix, aeternam moriens famam, Caieta, dedisti'.

En effet ce vers, une fois qu'on a enlevé le développement sur les enfers qui se trouve au milieu, se rattache aux funérailles de Misène: le cours du poème, 
s'écartant de celles-ci, y revient et fait que la narration redevient intacte, si bien qu'il ${ }^{17}$ a dit 'toi aussi' (tu quoque) à juste titre. Cette affirmation apparaîtra de manière plus claire si nous rappelons le point à partir duquel il y a eu écart : 'Quant au pieux Énée, il lui élève un tombeau à la masse imposante, avec les armes propres à ce héros, la rame et la trompette, sous le mont Aerius ${ }^{18}$ que, maintenant, on appelle Misène d'après ce personnage, et qui garde, pour toujours, ce nom au fil des siècles.' Cela aurait dû être suivi de 'Toi aussi, nourrice d'Énée, tu as donné en mourant une renommée éternelle à nos rivages'. Ainsi donc, les vers intermédiaires sur les enfers ont divisé ce qui aurait dû être un. Mais en fait, maintenant aussi on a cette unité, puisque le poète est revenu au point d'où il se souvenait être parti. C'est donc à juste titre que, liant les vers présents aux vers placés plus haut, il dit : 'Toi aussi, nourrice d'Énée, tu as donné en mourant une renommée éternelle à nos rivages.'

6 Tout est bien qui finit bien (Sed et nunc unum est... Ergo recte ait) dans la mesure où, du point de vue de TCD, par l'adverbe quoque, Virgile a bel et bien refermé la boucle de son récit, renoué avec le fil principal de ce dernier, qui en ressort intact et un: le commentateur ne se voit pas contraint de vraiment critiquer Virgile - ce serait exceptionnel -, qui a pensé à tout (ou du moins n'a-t-il rien oublié : meminerat poeta).

Ce passage du commentaire fait néanmoins question, à plusieurs titres : pour finir, TCD loue peut-être Virgile, mais les formulations de désapprobation - presque de regret - par lesquelles il est passé, dans une analyse qui plus est insistante, sont assez rares pour être relevées (sequi debuit, unum esse oportuit) ${ }^{19}$. D'ailleurs, l'idée que le poème devrait former une narration "une" (continue, sans digressions superflues) n'est pas une idée que TCD défend absolument dans l'ensemble de son commentaire : il connaît les mérites et même la nécessité de la uarietas, et la lassitude qu'est susceptible de faire naître, si elle reste ininterrompue, la continuatio narrationis ${ }^{20}$. Mais c'est en fait le statut même de la catabase qui est en cause : l'analyse de TCD tend, dans tous les cas, à en faire l'équivalent d'une comparaison, d'une description, d'une scène divine - en somme, d'un élément d'ornement poétique - et elle la désigne comme une digression (voir les termes transcrits en gras dans le passage cité) dont le caractère nécessaire n'est pas souligné ${ }^{21}$. Il me semble que s'exprime ainsi une forme de réticence vis-à-vis d'un passage de l'Énéide qui, de façon extrêmement surprenante, n'apparaît donc pas comme aussi essentiel que nous pouvons le considérer et qu'il a été en général considéré22, et à propos duquel TCD était peut-être moins à l'aise pour mettre en œuvre sa pratique propre du commentaire.

Que retrouve-t-on de celle-ci dans les interpretationes développées sur le chant 6 ? Et dans quelle mesure ce que nous venons de voir et qui n'est exprimé qu'a posteriori - au début du commentaire du chant 7 - se manifeste-t-il dans les analyses proposées par TCD?

\section{Une lecture globalement sans surprises : rhétorique et morale}

Rien ne laisse en fait présager, même dans le commentaire au début de la catabase, les remarques étonnantes de TCD au début du chant $7^{23}$. L'objectif principal du commentateur demeure toujours le même : montrer avec quel art Virgile loue Énée et avec quel art aussi il construit sa défense toutes les fois qu'il le faut -c'est le cas, en l'occurrence, lors des retrouvailles avec Palinure (dont la mort n'est pas due à l'incurie d'Énée) et, encore plus, Didon (Énée plaide ici coupable). Les discours qui occupent une large part ou la totalité de ces deux scènes (de Palinure dans un cas, d'Énée dans l'autre) 
sont ceux qui font l'objet de la lecture rhétorique la plus précise au sein du chant, l'un étant identifié à une petitio (une requête), l'autre faisant une place importante à la purgatio (la justification, en l'occurrence, du héros par lui-même) ${ }^{24}$. De fait, de façon plus générale, toutes les fois qu'il y a discours, TCD est, comme à son habitude, à son aise. Seulement, et de façon cohérente par rapport à ce que nous avons vu de ce qu'il pense de la catabase, il apparait comme particulièrement vigilant à l'endroit de tout ce qui pourrait donner une impression de superflu dans le superflu.

C'est le cas devant la première série de questions qu'Énée adresse, ici (Aen. 6, 318-320, aux abords du Styx) à la Sibylle :

'Dis-moi, ô vierge, que signifie ce rassemblement au bord du fleuve? Que veulent ces âmes ? Comment se fait le partage entre celles qui quittent les rives et celles qui de leurs rames balaient les eaux couleur de plomb?'

Après avoir souligné, comme souvent, que la question vaut en fait présentation (propositio ) du discours à suivre pour autant qu'elle en définit le plan, TCD relève breuiter qui caractérise, au vers suivant, la réponse de la Sibylle, et souligne (I. V. I, p. 551, p. 12-14) :

'breuiter' quippe loqui debuit relatura superflua, ne quae fuerant necessario agenda morando differret

elle se devait en effet de parler 'brièvement' (breuiter), vu qu'elle allait rapporter des informations superflues, pour ne pas différer en les retardant les actes qu'il était indispensable d'accomplir.

Après une apostrophe élogieuse en guise d'introduction, la Sibylle surprend (TCD en tout cas) en ne répondant pas à la première des questions d'Énée, mais en ajoutant une information supplémentaire, sur l'identité des lieux (c'est le v. 323: «tu vois l'étang profond du Cocyte, tu vois le marais du Styx »). Mais la Sibylle, affirme tout de suite le commentateur, a bien fait d'élargir ainsi le sujet, sans perdre de vue la brièveté : il eût été plus dommageable pour le récit et, à la base, pour l'action des deux héros pressés, qu'Énée, insatisfait par cet échange, fût amené à poser une nouvelle question (I.V. I, p. 551, 1. 20-26). Un raisonnement similaire est appliqué à la réponse qu'Anchise fait à Énée à propos du jeune Marcellus (I. V. I, p. 615, 1. 28-p. 616, 1.4) : la réponse excède la question pour qu'Énée n'en demande pas davantage ${ }^{25}$.

Parfois, le surplus d'informations est reconnu, à demi-mots, comme nécessaire : c'est le cas à propos du début de la présentation que la Sibylle fait du Tartare, après l'apostrophe introductive et une précision sur la source de ses informations (Hécate elle-même : Aen. 6, 564-565). Si la Sibylle répond "pleinement" aux questions d'Énée, en incluant des données qu'il n'avait pas demandées, ce peut être pour garantir l'utilité de cette réponse (I.V. I, p. 583, 1. 9-11):

car elle eût décrit vainement les faits et gestes propres au lieu, si elle n'avait pas commencé par présenter l'enquêteur et juge, en charge des crimes et des châtiments ${ }^{26}$.

Une telle tolérance donne à penser que TCD reconnaît l'importance singulière de la description du Tartare... puis de l'Élysée. La valeur morale de ces passages qui forment le cœur du "cœur" ne le laisse, effectivement, pas indifférent: en ces lieux, Virgile a spécialement dit «ce que c'est que de vivre mal» et présenté les criminels et leurs châtiments, avant de montrer « en quoi le fait d'avoir vécu selon le bien était utile après la vie » (I. V. I, p. 591, 1. 2-24).

Le passage où TCD s'étend le plus sur cet aspect de l'enseignement de Virgile en ce chant est le passage si essentiel où la Sibylle interrompt sa description des crimes et des 
châtiments ${ }^{27}$. Quoique le commentateur n'ait pas la tête au genre épique, il semble en saisir l'aspiration essentielle à un "tout dire" qui se heurte nécessairement à la frontière entre dicible et indicible ${ }^{28}$. Le détail du topos des nombreuses bouches - puisque c'est de lui qu'il s'agit - ne l'intéresse pas, mais voici ce qu'il dit alors que la Sibylle a déjà mis un frein à son énumération par une formule résomptive (ausi omnes immane nefas ausoque potiti, «tous ont osé un crime abominable et se sont rendus maîtres du fruit de leur audace ", Aen. 6, 624):

Iam specialis narratio producta per multos faceret maiorem properantibus moram: confugit ad generalitatem dicens AVSI OMNES IMMANE NEFAS AVSOQUE POTITI : 'omnes', inquit, isti nefariorum scelerum rei sunt et quicquid adgressi sunt perfecerunt. Cum dicit 'omnes', et illos tetigit quos designatis criminibus posuit. Omnia haec poenarum et criminum genera Vergilius non inaniter posuit; neque enim id curauit ut ostenderet se curare actus inferos, sed ut suppliciorum metu ad bonos mores singulos quosque conuerteret et apertius docti rectius uiuerent, certi deterius puniri post mortem quae sciendo uindicauerint homines aut praetermiserint interrogando.

À partir de là, si la narration s'était prolongée au travers des cas spécifiques de nombreux hommes, elle eût accru le retard qu'ils avaient, malgré leur hâte. Il a eu recours à la généralitée ${ }^{29}$ en disant 'tous ont osé un crime abominable et se sont rendu maîtres de ce qu'ils avaient osé' : 'tous' ces individus, dit-il, sont coupables d'infâmes méfaits et ils ont accompli jusqu'au bout ce qu'ils ont entrepris. Quand il dit 'tous' (omnes), il fait référence aussi à ceux qu'il a mentionnés en désignant leurs crimes. Mais Virgile n'a pas mentionné tous ces genres de crimes et de châtiments en vain, et il n'a pas eu le souci de montrer qu'il se souciait de ce qui est fait aux enfers, mais, par la crainte des supplices, de convertir tout un chacun aux bonnes mœurs, de manière à ce que, plus clairement instruits, les hommes vivent de manière plus juste, une fois assurés qu'après leur mort, une punition pire frapperait ce que les hommes, en connaissance de cause, auraient vengé, ou ce qu'ils auraient négligé au cours de leurs enquêtes ${ }^{30}$.

Dans un premier temps, une forme d'empressement à tout dire - en l'occurrence, à dire tous les crimes et les châtiments - est reconnue à Virgile, l'important étant cependant de souligner qu'il ne s'est pas laissé ainsi porter par un réel intérêt pour "l'actualité" infernale ; le but du poète a plutôt été de susciter ou de nourrir la crainte des châtiments de manière à amener les hommes à vivre de manière plus juste. Dans un deuxième temps, cependant, quand apparait le topos des nombreuses bouches (soit, pour TCD, une autre formule de généralisation, generalitas), le commentateur assume plutôt l'idée que Virgile n'a pas tout dit ni même voulu paraître tout dire : l'immensité du sujet est soulignée conformément au sens premier du topos et à la propriété de l'épopée qu'il désigne -, mais TCD identifie en outre une stratégie plus subtile, de la part du poète, pour agir sur les esprits et les mœurs des hommes.

Mire tamen addidit non, mihi si lingvae centvm sint oraqve centvm, ferrea vox, omnis scelervm comprendere formas, omnia poenarvm percvrrere nomina possim : generalitas enim necessarie adiecta est, ne poeta uideretur omnia descripsisse quae apud inferos gerebantur, quoniam fuit impossibile latitudinem tantam plene complecti, uel ne contemptui haberentur poenae propositae ex cotidiana meditatione ; amplius enim supplicia incerta metuuntur. Certe etiamsi sola fuerant quae dicta sunt, accessit humanis actibus quod prodesset ex poetae mendacio. Plus enim fecit timere et minus peccare metuentem, eoque res redit ut uiuatur rectius cum infinita formido est.

Il a cependant ajouté de manière étonnante: 'Non, si j'avais cent langues, cent bouches, une voix de fer, je ne pourrais énumérer toutes les formes de crimes, passer en revue tous les noms des châtiments'. Cette formule générale a été ajoutée 
de manière nécessaire : le poète ne devait pas paraître avoir décrit tout ce qui se passe aux enfers, parce qu'il est impossible d'embrasser complètement une matière si vaste, ou pour éviter que ne soient tenus en mépris les châtiments que l'on se représente à la faveur d'une méditation quotidienne ; en effet on redoute davantage les supplices s'ils restent imprécis ${ }^{31}$. En tout cas, même s'il y avait eu seulement les supplices évoqués, s'est ajouté aux actes des hommes ce qui pouvait être utile dans le mensonge du poète. Il a accru la peur et limité les fautes en suscitant la crainte, et le résultat est que l'on vit de manière plus juste quand l'effroi est sans limites.

Ainsi, Virgile aurait interrompu son énumération et ajouté une expression qui accentue le caractère incomplet de celle-ci, pour agir de manière encore plus efficace sur l'esprit des hommes et, concrètement, pour accroitre la crainte des supplices qu'ils pouvaient avoir d'après ce qu'ils en connaissaient dans le monde des vivants. Avec l'image des cent bouches, Virgile laisse planer un doute, et même crée un suspens effroyable en forçant les hommes à s'imaginer des châtiments encore plus nombreux et sans doute inédits : dans la présentation qu'il en a faite, le poète a déjà exploité les ressources propres de son art - en l'occurrence, sa capacité au "mensonge" - en ne se contentant pas de refléter les usages réels des hommes. Je comprends que TCD pense ici à l'enchevêtrement des exemples mythologiques et "historiques" propre à la description virgilienne du Tartare ${ }^{32}$. Je mesure aussi que la manière dont il s'exprime ici ne lui est pas familière : la notion de "mensonge poétique" fait son unique apparition à cet endroit dans les $I . V^{33}$; et le passage comporte des échos possibles à des textes et des pratiques chrétiennes ${ }^{34}$. Il semble que TCD parle ici un langage différent du sien, de façon à proposer cependant, surtout à propos de la generalitas des v. 625-627 (les nombreuses bouches), une interprétation originale suivant la perspective de sa propre lecture morale du poème.

\section{Du deuil à la joie : un regard singulier sur la richesse et la fonction d'Énéide 6}

La tension observée entre une certaine tendance de Virgile à l'exhaustivité et l'impossibilité de tout dire (complétée à l'occasion par le pouvoir de suggestion d'une parole qui ne dit pas tout) s'exprime de nouveau à la fin du chant, à propos du second discours d'Anchise. Une distinction se fait entre la présentation détaillée et complète qu'Anchise doit à son fils pour l'instruire (p. 605, 1. 9-13, avec, en particulier, 1.12-13: oportuit enim eum, ut instructiorem filium redderet, cunctos ex ordine numerare $)^{35}$ et le nécessaire abrègement auquel a procédé Virgile : il n'aurait pu tout faire entrer dans son poème et il aurait eu tort de le faire (I. V. I, p. 617, 1.13-16). Comme pour la présentation des criminels du Tartare, «il s'est tourné vers une expression générale " ( C'est ainsi qu'ils vont, çà et là, par la région entière, dans les vastes plaines de l'air, et ils parcourent tout du regard", Aen.6, 886-887), generalitas à laquelle est ici reconnu le pouvoir de signifier sans dire en évitant les délais (totum, etiam non dictum potuit significare omni mora submota, I. V. I, p. 617, p. 16-18). Il y a cependant une omission qui est signalée et qui, s'il avait pu la respecter parfaitement, eût été tolérée et louée de la part du père d'Énée : c'est celle des tristia, des lugenda, dans un discours - le discours dédié à la Parade des héros qui eût dû n'être fait que de laeta pour insuffler ce seul sentiment à Énée.

Cela vaut, en fait, pour l'ensemble des deux discours d'Anchise : lorsque le premier, sur le sort des âmes, est amené par une question d'Énée « qui ne sait pas » (inscius), TCD a une remarque générale sur le système de questions-réponses qui lui semble caractéristique de 
cette section du poème et qui oriente même ce dernier dans un sens particulier (I. V. I, p. 600, 1.15-18).

Per interrogantis respondentisque personam eo poetae ducitur carmen, ut Aeneas laetus ab inferis dimittatur et felicitas eius appareat, cui soli contigit, antequam nascerentur nosse posteros suos.

Par la personne de celui qui pose des questions et de celui qui répond, le poème est conduit de telle sorte qu'Énée soit renvoyé joyeux des enfers et que sa chance soit manifeste, lui, le seul à qui il ait été donné de connaître ses descendants avant même leur naissance.

Eo... ducitur... ut n'est pas aisé à traduire, mais semble bien exprimer l'idée d'un sens (direction et signification) que le dispositif propre à cette partie finale (ce jeu de questions-réponses que TCD rapproche plus loin des dialogues) confère enfin à tout le chant : ce dernier révèle à quel point Énée est unique (et d'autant plus louable), mais surtout, il vise à exalter le héros en lui faisant connaître le futur glorieux dont il est porteur.

21 La joie n'est certes pas l'émotion ou le ton que l'on associerait spontanément au chant 6 . De fait, ce qui domine d'abord, aussi dans le commentaire de TCD, c'est un climat de deuil au sein duquel l'expression de la miseratio reçoit, à plusieurs reprises, une attention certaine $^{36}$. Ce que souligne, alors, la remarque du commentaire au tout début des discours d'Anchise, c'est un changement de direction, reposant sur un changement de ton, qui aurait dû être observé parfaitement jusqu'à la fin du chant. Si tel n'est pas le cas, cela est dû à Énée qui, à la vue du jeune Marcellus triste, n'a pas pu s'empêcher d'adresser à son père des questions à son sujet. Anchise pleurant regrette de devoir répondre (I. V. I, p. 615, 1. 29-616, 1. 1, à propos de ingentem luctum ne quaere tuorum, v. 868) :

'je voudrais, mon fils, que tu ne t'enquières pas des sujets de larmes et je ne voudrais pas que tu connaisses les malheurs des tiens : ils pourraient briser ton courage'.

Le fait est que le second discours d'Anchise a pris très vite, aux yeux de TCD, une forme exhortative, et que son but propre est de renforcer le courage d'Énée (I. V. I, p. 608, 1. 7-9; p. 611, 1. 2-3 ; p. 613, 1.18-19; p. 618, 1. 5). Pour ne pas risquer de l'affaiblir, Anchise aurait donc, jusqu'au bout, continué à omettre les tristia (I. V. I, p. 617, 1. 26-28), comme il l'avait fait, au début, en ne parlant pas directement à Énée de sa propre mort, mais en lui annonçant plutôt la naissance d'un fils "posthume" (I. V. I, p. 606, 1. 14-18). L'attention prêtée par TCD à cette tonalité « optimiste » du discours d'Anchise est intéressante pour la compréhension - et la réception - du chant 6 dans son ensemble, mais elle l'est aussi plus précisément, s'agissant de Marcellus, pour la réflexion qu'elle conduit à avoir sur le bien-fondé d'un éloge funèbre dont on sait qu'il ne laissa pas indifférente la famille d'Auguste $^{37}$. Certaines représentations figurées de la scène de la recitatio d'Énéide 6 par Virgile devant Auguste font voir un Virgile effarouché tel qu'en lui-même ${ }^{38}$, sans doute, mais peut-être aussi parce qu'il voit Auguste courroucé après l'évanouissement d'Octavie 39 .

\section{Conclusion}

Primo laeta narrauit filio, praetermisit tristia, solum unum quod esset dolendum de Marcelli rettulit et hoc ipsum non uoluntate, sed ut interroganti Aeneae responderet. Cetera ei sub generalitate dixit ostensa nec dixit specialiter quae. Quando enim tantum camporum uel tantam multitudinem animarum sermone posset includere ${ }^{40}$ ? 
Il a d'abord raconté des choses joyeuses à son fils, laissant de côté les motifs de tristesse: il n'a rapporté qu'un seul événement susceptible de faire souffrir, à propos de Marcellus, et encore n'a-t-il pas fait cela de sa propre volonté, mais pour répondre à Énée qui l'interrogeait. Quant au reste, il dit de manière générale que cela lui a été montré sans dire spécifiquement de quoi il s'agit. Quand, en effet, aurait-il pu faire entrer dans son propos des espaces si étendus et un nombre, si immense, d'âmes?

Le commentaire que TCD fait des derniers vers - juste avant la sortie - où le poète abrège le discours d'Anchise et en résume les développements ultérieurs, exprime une dernière fois pour ce chant l'admiration que le commentateur nourrit pour la breuitas de Virgile, qui sait s'arrêter quand il faut, passer du particulier au général, aller, alors, droit au but ${ }^{41}$. Et en même temps, il exprime, sous la forme d'une interrogation rhétorique mais avec deux formes de tantus qui suggèrent une exclamation, qu'il est bien conscient de la richesse du sujet dont s'est emparé Virgile. TCD aurait peut-être plus complètement rendu compte de cette richesse s'il avait mené à bien son projet de treizième livre consacré aux realia et aux antiquités contenus dans l'Énéide ${ }^{42}$ : en particulier dans le cas du commentaire du chant 6 , si conscient soit-on du fait que TCD n'est pas Servius, et que son commentaire est essentiellement rhétorique, on peut ressentir un manque quand les histoires mythologiques ou l'évocation des figures historiques font seulement l'objet d'une reformulation. Au sein de ce commentaire même, le traitement que TCD fait de la catabase ne se signale pas, cependant, par un manque d'attention, comme aurait pu le causer ce livre si plein de mythes et pour beaucoup, de vérités ou de sagesse; TCD ne fléchit pas dans sa lecture littérale et dans une «interprétation » (interpretatio qui tend à la réécriture) où, ici comme ailleurs, il ne se sent pas enfermé par la lettre exacte du texte. La mise entre parenthèses - sans doute théorique et temporaire - de la catabase qu'il propose au début du chant 7 , à propos de $T u$ quoque continuera ainsi de surprendre, à moins qu'elle ne trahisse la difficulté que ce commentateur si singulier a eue pour tenir son propre cap face à ce « labyrinthe du texte ${ }^{43}$ ».

\section{BIBLIOGRAPHIE}

BRÉCHET Chr., CLÉMENT-TARANTINO S., « La poikilia chez Homère et la uarietas chez Virgile d'après les scholies antiques », dans H. Vial (éd.), La Variatio. L'aventure d'un principe d'écriture de l'Antiquité au XXIe siècle », Paris, Classiques Garnier, 2014, p. 21-43.

CLÉMENT-TARANTINO S., « Le discours d'Ilionée au livre VII de l'Énéide (VII, 213-248) », Exercices de rhétorique [en ligne], 2, 2013 (mis en ligne le 18 novembre 2013). URL : http:// rhetorique.revues.org/181; DOI : 10.4000/rhetorique.181

COURCELle P., « Les Pères de l'Église devant les Enfers virgiliens », Archives d'histoire doctrinale et littéraire du Moyen Âge, 30, 1955, p. 5-70.

COURCELLE P., Lecteurs chrétiens et lecteurs païens de l'Énéide, Paris, de Boccard, 1984, 2 vols.

DE PROOST P.-A., « Ficta et facta. La condamnation du 'mensonge des poètes' dans la poésie latine chrétienne », Revue des études augustiniennes 44, 1998, p. 101-121. 
DAGHINI A., « La breuitas nelle Interpretationes Vergilianae di Tiberio Claudio Donato », in F. Stok (dir.), Totus scientia plenus. Percorsi dell'esegesi virgiliana antica, Pisa, Edizioni ETS, 2013, p. 401-428.

DEREMETZ A., « Virgile et le labyrinthe du texte », Uranie 3, 1993, p. 45-67.

FORD A., Homer. The Poetry of the Past, Ithaca-London, Cornell University Press, 1992.

GIOSEFFI M., « Ritratto d'autore nel suo studio. Osservazioni a margine delle Interpretationes

Vergilianae di Tiberio Claudio Donato ", in M. Gioseffi (ed.), E io sarò tua guida. Raccolta di saggi su Virgilio e gli studi virgiliani, Milano, 2000, p. 151-215.

GIOSEFFI M., « Un libro per molte morali: osservazioni a margine di Tiberio Claudio Donato lettore di Virgilio ", dans I. Gualandri, F. Conca, R. Passarella (dir.), Nuovo e antico nella cultura greca-latina di IV-VI secolo, Milano, 2005, p. 281-305.

GOSSEREZ L., Poésie de lumière : une lecture de Prudence, Louvain-Paris-Sterling, VA, Peeters, 2001. HARDIE P., " In the Steps of the Sibyl : Tradition and Desire in the Epic Underworld », Materiali e Discussioni per l'analisi dei testi classici 52, 2004, p. 143-156.

HARDIE P., The Last Trojan Hero. A Cultural History of Virgil's Aeneid, London-New York, I.B. Tauris. HINDS S., Allusion and Intertext. Dynamics of Appropriation in Roman Poetry, Cambridge, Cambridge University Press, 1998.

JEANJEAN B., " Virgile au secours de la Correspondance de Jérôme », dans P. Laurence et F. Guillaumont (éds.), Les écritures de la douleur dans l'épistolaire de l'Antiquité à nos jours, Presses Universitaires François Rabelais, 2010, p. 133-150, nouvelle édition en ligne (générée le 2 mai 2014), http://books.openedition.org/pufr/2214.

MARSHALL P.K., « Tiberius Claudius Donatus on Virgil Aen. 6.1-157 », Manuscripta 37 (1), 1993, p. 3-20.

MOST G., « Memory and Forgetting in the Aeneid », Vergilius 47, 2001, p. 148-170.

PIROVANO L., Le Interpretationes Vergilianae di Tiberio Claudio Donato. Problemi di retorica, Roma, Herder, 2006.

PIROVANO L., « Tiberio Claudio Donato e i 'progymnasmata' ", Incontri Triestini di Filologia Classica 7, 2007-2008, p. 177-199 (accessible en ligne à l'adresse : http://www2.units.it/polymnia/calamo/ Calamo2008_PIROVANO.pdf)

PIROVANO L., « Cursim scripsi quae potui : Tiberio Claudio Donato rilegge il suo 'libro' », CentoPagine IV, 2010, p. 135-146 (accessible en ligne à l'adresse : http://www2.units.it/polymnia/ iniziative/SCA2010_PIROVANO.pdf)

PIROVANO L., « Note filologiche al ‘nuovo' Tiberio Claudio Donato », in F. Stok (dir.), Totus scientia plenus. Percorsi dell'esegesi virgiliana antica, Pisa, Edizioni ETS, 2013, p. 341-360.

VALLAT D., « Le commentaire de T. Claude Donat au chant 1 de l'Énéide, sa place dans les débats virgiliens et ses relations avec Servius », Eruditio Antiqua [en ligne] 1, 2009, p. 155-184.

ZETZEL J., « Romane memento : Justice and Judgment in Aeneid 6 », Transactions of the American Philological Association 119, 1989, p. 263-284. 


\section{NOTES}

1. Ce texte a pour origine une intervention présentée lors du Symposium Cumanum dédié à la réception d'Énéide 6 dans l'histoire culturelle (« Aeneid Six and its Cultural Reception », 25-27 juin 2013, org. Bill Gladhill). La réception dont ce chant a été l'objet atteste de fait son statut exceptionnel, eu égard, en particulier, aux interprétations si nombreuses et variées auxquelles il a donné lieu. Au sein d'une bibliographie immense, on mentionnera en particulier les travaux de P. Courcelle (COURCELLE 1955 et 1984) et, plus récemment, le chapitre 2 de HARDIE 2014.

2. C'est à Lucrèce que l'on pense en particulier face aux monstres séjournant dans l'entrée des enfers, surtout lorsque la « docte » Sibylle dissuade Énée d'essayer de les combattre parce que ce ne sont que des «vies sans corps", voletant «sous l'apparence creuse d'une forme " (Aen.6, 292-294).

3. Sur ce point, voir en particulier ZETZEL 1989.

4. C'est ainsi que Servius prend fama est employé, au début du chant, à propos du vol de Dédale : ad Aen. 6, 14.

5. [uult autem intellegi] falsa esse omnia quae dixit (Servius, Commentaire sur l'Énéide 6, ad Aen. 6, 893).

6. Je me réfère à l'Énéide traduite par Paul Veyne : VEYNE 2012 et VEYNE 2013. Dans la préface de chaque édition, le savant procède à un certain nombre de mises au point très utiles à propos de l' Énéide, réagissant à des défauts qui lui étaient tacitement imputés depuis de nombreuses années et qui étaient sans doute pour beaucoup dans le désintérêt où elle était tenue ; certaines de ses vues sur le poème sont cependant tout à lui, comme le désormais fameux « l'Énéide est amusante à lire » (VEYNE $2012: 9$ ).

7. Dans la note citée (ad Aen. 6, 893), Servius introduit la remarque que j'ai reproduite en disant que c'est le sens «clair» (apertus) «du point de vue de la poésie» (poetice); et c'est à la signification physiologique des deux portes qu'il s'intéresse ensuite.

8. D. Vallat a le plus récemment repris le dossier des rapports entre le commentaire servien et les Interprétations virgiliennes: VALLAT 2009. Les travaux fondamentaux sur Tiberius Donat sont: SQUILLANTE-SACCONE 1985 ; GIOSEFFI 2000 ; PIROVANO 2006. Je vais citer le texte des Interpretationes Vergilianae d'après l'édition de GEORGII pour Teubner (1905-1906), en indiquant à chaque fois le volume, la page et la ou les lignes.

9. Voir surtout Macrobe, Saturnales, I, 24, 13 : «Ne souffrons pas que les mystères du poème nous restent fermés, mais, en cherchant à pénétrer le sens qui s'y trouve caché, nous entr'ouvrirons le sanctuaire pour l'offrir au culte des savants » (traduction H. BORNECQUE, Classiques Garnier); la constante profondeur du poème est régulièrement soulignée au livre III (III, 2, 7 ; III, 7, 1...).

10. Sur la breuitas dans les Interpretationes Vergilianae, voir spécialement DAGHINI 2013.

11. Voir le proème des I.V. I, p. 4, 1.24-p.5, 1.22 : expliquant à son fils ce qu'il pourra retirer de l'étude de Virgile, TCD identifie d'abord le poète au plus grand rhéteur (rhetorem summum, I, p. 4, 1. 26), puis il en fait un spécialiste de toutes les disciplines et un maitre de vie et de morale, dont peuvent apprendre aussi bien les pères que les fils, les maris et les femmes, les généraux et les soldats, les bons citoyens, les prêtres, ceux qui recherchent des amitiés intactes... (cf. I.V. I, p. 5, 1. 7-20).

12. L'expression est employée dans le passage cité dans la note précédente (I.V. I, p. 5, 1.4-7 : laudabis eum cui licuit uniuersa percurrere, qui se diuersae professionis et diuersarum sectatoribus artium beniuolum praebuit peritissimumque doctorem) : c'est Virgile comme un autre Homère, sauf que la référence n'est pas explicitée (elle ne l'est pas dans ce commentaire).

13. Dans le proème du commentaire, TCD prend la défense de Virgile contre ses détracteurs, en particulier à propos des incohérences reprochées au poète. Pour TCD, celles-ci s'expliquent par l'attention que Virgile porte, en chaque circonstance, à ce qu'exigent celle-ci, et / ou la personne 
qui parle, le lieu, le moment... Le poète n'assène pas des vérités, et si l'on considère la façon dont il présente son sujet au début de l'Énéide, «nous trouverons», écrit TCD, «que Virgile a seulement déclaré son intention de retracer la geste d'Énée, et non de faire sienne, comme s'il en garantissait la vérité, quelque partie d'une science cachée ou de la philosophie » (I.V. I, p. 6, 1. 9-12). L'expression scientia interior qui est alors employée apparaît dans les Homélies sur le Lévitique d'Origène dans la traduction de Rufin (Homélies I, 4, 1.15 : l'édition utilisée est celle de M. BORRET pour les Sources chrétiennes, Origène. Homélies sur le Lévitique, I, Paris, Le Cerf, 1981) : il s'agit de la «science intérieure » du verbe de Dieu qui s'obtient lorsqu'on lève le voile sur les mots, comme le prêtre enlève la peau du jeune taureau offert en holocauste de manière à en mettre à nu les « membres intérieurs ».

14. Par contraste, TCD n'a pas été arrêté par l'emploi de Caieta comme nom géographique dès la fin du chant 6 , précisant simplement que le nom se comprend mieux en référence à la persona du poète vu qu'«Énée n'avait pas encore attribué ce nom au lieu » (I.V., p. 619, 1. 4-5). Cf. Servius ad loc.

15. L'épitaphe de Misène s'achève au v. 235 et l'invocation qui signe le début de la catabase commence au v. 296; dans le passage intermédiaire, Énée a déjà pénétré dans la caverne profonde où il va s'engouffrer avec sa guide après l'accomplissement des sacrifices dus.

16. La portion de texte concernée est remarquable par sa nature et son contenu, mais aussi par son étendue: dans l'analyse similaire que l'on trouve en I.V. II, p. 290, 1.28-p. 291, 1.5 (pour résoudre l'apparente contradiction contenue dans abnueram bello Italiam concurrere Teucris en Aen $.10,8$, TCD relit de près les déclarations de Jupiter dans la prophétie du chant 1 ), il ne s'agit que de deux vers et demi (Aen. 1, 260b-262 qui morcellent la narratio futurorum et qu'il suffit de retrancher pour avoir l'enchaînement sublimem feres ad sidera caeli magnanimum Aenean [v. 259-260a]; bellum ingens geret Italia [263])!

17. « Il » désigne bien sûr Virgile. J'ai pour habitude de conserver dans la traduction la relative indétermination qui est récurrente et caractéristique dans les commentaires de Servius et de TCD au moins, quant au sujet des actions ayant trait à la composition poétique.

18. C'est ainsi que comprend TCD : voir I.V. I, p. 540, 1. 32-p. 541, 1. 6.

19. Il n'y a rien de tel dans le passage du livre 10 mentionné précédemment (note 16), rien non plus dans celui du livre 12 où TCD observe que l'histoire de Iapyx, intercalée (interposita), a divisé la narration (I.V. II, p. 599, 1. 30-p. 600, 1. 1). On sent un peu plus de réserve en ce qui concerne la description du monstre Rumeur (Fama) au chant 4, parce que l'hyperbate (I.V. I, p. 377, 1.14) à laquelle, en tant que description interposée, elle donne lieu selon le commentateur, «induit en erreur ceux qui sont moins attentifs » (p. 377, 1. 15-16); il semble qu'une « narration une » eût été ici préférable (hanc si subtrahas, erit una narratio, I.V. I, p. 379, 1. 21). D'une manière plus usuelle et générale, ce qui peut paraître superflu dans la narration de Virgile est justifié par TCD soit parce que, en réalité, c'est nécessaire (c'est le cas des scènes divines entre Vénus et Jupiter, Jupiter et Mercure au chant 1: voir I.V.I, p. 66, 1.7-8, 1.27-32, p. 67, 1.16-2 avec completis igitur quae necessario uidentur interposita), soit parce que c'est le chemin à suivre vers ce qui est nécessaire ( I.V. I, p. 303, 1. 2-5, p. 337, 1. 8-9, avec la généralisation suivante : more suo multa superflua descripsit, ut ad eum locum ueniret qui fuerat necessarius).

20. C'est un aspect que j'ai étudié, sous l'angle de la uarietas, dans BRECHET-CLÉMENT-TARANTINO , 2014 : 39-42. Dans le commentaire du chant 6, figurent plusieurs exemples, comme la remarque sur la prétérition du v. 601 : Quid memorem Lapithas, Ixiona Pirithoumque? TCD l'analyse comme une forme de transition (transitus) vouée «à éviter que la continuité de la narration ne devienne horrible ». De même, à propos de la transition du v. 854, à l'intérieur du discours d'Anchise (Sic pater Anchises atque haec mirantibus addit) : « il y a des transitions qui servent à éviter l'ennui d'une narration une et continue» (transitus sunt quidam, ne una et continua narratio taediosa sit). Il me semble que TCD chérit tout de même l'idée d'une Énéide dont la narratio - à prendre au sens rhétorique, le projet de lecture de TCD tendant dès le début à faire de l'Énéide un discours d'éloge 
et de défense d'Énée : voir I.V. I, p. 2, 1. 7-25 - se réduirait (encore plus) à l'essentiel, totalement « une et continue ». C'est un aspect que je compte étudier dans le détail très prochainement, de manière à faire apparaître, en sens inverse (ou de manière complémentaire), ce que TCD impute ou reconnaît de manière irréductible au poème virgilien (notamment, la recherche de l'ornatus, à travers les comparaisons, certaines descriptions...).

21. À la différence du Conseil des dieux du chant 1, pour reprendre un exemple donné supra, note 16. Sur l'importance de la necessitas, DAGHINI 2013 : 416-417.

22. Virgile l'a rendu essentiel, par la richesse de son contenu, le « désir » et le plaisir suscités par sa narration, lui évitant ainsi d'apparaître comme une sorte d'appendice pour le reste du poème : cf. HARDIE 2004 : 144 en particulier. Certains critiques ont insisté sur le fait que le chant 6 n'était pas intégré à l'intrigue au point d'apparaître comme un maillon nécessaire pour les les chants 7-12: « No narrative thread links it to any later event, in terms of plot, it could vanish completely without making any difference at all » (MOST 2001 : 170). Le chant 6 est tellement marqué par la nécessité de faire vite, de ne pas prendre (trop) de retard, qu'il peut d'ailleurs en venir à se désigner a contrario comme un haut-lieu de la mora, du délai indispensables à la longueur épique ; TCD partage, pour ainsi dire, avec la Sibylle (voir notamment Aen. 6, 37 et 6, 539 - l'adverbe breuiter est d'ailleurs employé trois fois au chant 6 et caractérise toujours une réponse de la prêtresse), la conscience d'une telle nécessité : voir I.V. I, p. 537, 1. 11 sqq.

23. Rien ne le fait attendre non plus dans le début du commentaire du chant 6 (ce qui concerne les v. 1-157), découvert à la fin du XX ${ }^{\mathrm{e}}$ siècle par P. K. Marshall : MARSHALL 1993 ; cf. PIROVANO 2013. 24. Le commentaire de la rencontre entre Énée et Palinure commence en I.V. I, p. 553, avec la disculpation immédiate (1.9-13) d'Énée pour le fait que Palinure, comme Leucaspis et Oronte (1. 2-5), disparus dans la tempête du livre 1, n'a pas été inhumé. Le discours de Palinure est analysé comme une « requête » à partir du v. 365 (eripe me his, inuicte, malis) : I.V. I, p. 556. Sur la petitio dans le commentaire, voir CLÉMENT-TARANTINO 2013, où l'accent est cependant mis sur le discours d'Ilionée au chant 7. En ce qui concerne les "retrouvailles» d'Énée et de Didon, le commentaire s'en trouve de la p. 565 au haut de la p. 569 : Énée plaide coupable (p. 65, 1. 26 sqq., p. 567, 1. 26-28) parce qu'il ne lui serait pas possible de nier les faits - il a trahi Didon qui l'avait accueilli, avec ses hommes, et qui l'aimait -, et il invoque la volonté des dieux comme un cas de force majeure (maior uis : p. 567, 1. 12-17; voir PIROVANO 2006 : 143-144).

25. Le problème tient sans doute moins ici au retard supplémentaire que cela causerait à la narration, qu'à un ton - lugubre, endeuillé - que le discours d'Anchise n'aurait pas dû prendre. Je reviens sur ce point plus loin.

26. Il s'agit de Rhadamante, Aen. 6, 566-569.

27. D'autres morales du texte de Virgile qui traversent les I.V. et se retrouvent dans le commentaire du chant 6 concernent l'amitié, le bon citoyen et l'attachement à la patrie ( PIROVANO 2007-2008 : p. 4 prend l'exemple du commentaire que TCD fait du v. 621 - uendidit hic auro patriam, pour montrer comment ce commentateur va bien au-delà de la paraphrase - en l'occurrence, identifier hic ne l'intéresse pas, mais rappeler ce que signifie la patrie et préciser ce que signifie le fait de vendre sa patrie sont les objectifs d'une amplification rhétorique où il déborde largement du texte de Virgile).

28. L'examen auquel TCD soumet infandum au début d'Énéide 2 (Aen. 2, 3) est intéressant aussi en ce sens, que le terme ne s'explique pas seulement, pour le commentateur, en termes de gravité du sujet (d'intensité de la douleur du narrateur Énée), mais en termes d'immensité de ce sujet : il $\mathrm{y}$ a en effet une grande variété de douleurs (« légères, moyennes, très graves ", accumulées au gré d'infortunes nombreuses et variées et, à ce titre, indicibles : voir I.V. I, p. 146, 1.24-25). TCD n'est sans doute pas préoccupé par ce qu'il y a d'épique dans l'Énéide, mais il apparaît néanmoins comme extrêmement sensible à cet aspect essentiel du genre, son aspiration à l'exhaustivité. Sur ce point en rapport avec le topos des nombreuses bouches, je renvoie à la lecture par Andrew 
Ford de la version fondatrice du topos au chant 2 de l'Iliade : FORD 1992 : 57 sqq. et surtout 72 sqq. Sur les dynamiques de réécriture de ce même topos, voir spécialement HINDs 1998 :35-46.

29. La notion de generalitas et son antonyme, la specialitas, sont récurrents dans le commentaire. En rapport avec la breuitas, voir DAGHINI 2013 : 417-422.

30. Cf. ce qui est dit à propos des fonctions de Rhadamante en I.V. I, p. 583, 1. 20-22.

31. Les v. 574-575 («tu vois quel garde est assis dans le vestibule, quel être garde l'entrée?») étaient déjà lus de manière semblable : voir I.V. I, p. 584, 1.575-578. Cf. également I.V. I, p. 262, 1.1-3, où le départ d'Énée de Troie pour une destination inconnue (incerti quo fata ferant, Aen. 3, 7) est désigné comme un exemple de poena maxima, à cause de l'incertitude même ; une épreuve dont les contours sont un tant soit peu définis, poursuit alors TCD, peut être adoucie par une préparation sans relâche (meditatione continua).

32. Je renvoie à l'analyse précise de la description du Tartare dans ZETZEL 1989 : 265 sqq. Le savant commence par rappeler que Virgile combine deux types différents de description, l'un mythologique, le second philosophico-religieux («normally found in texts stemming from an Orphic-Pythagorean milieu », 266) ; j'ai dit « historiques » en référence à la couleur romaine que Virgile a donnée aux crimes relevant du second type, en permettant même au lecteur de penser à des figures historiques précises (ZETZEL 1989: 271). TCD ne s'adonne pas pour sa part à ce genre d'identification, mais s'en tient à la désignation et, éventuellement, à l'explicitation des «vrais » crimes qui sont énumérés (voir par exemple I.V. I, p. 588, 1. 1-13, à propos des v. 608-613).

33. Voir notamment DE PROOST 1998. Telle que je comprends la remarque qui fait intervenir cette notion de mensonge des poètes, elle réfère moins à un voile qu'il faudrait ôter pour comprendre ce que Virgile a dit sur la nécessité de vivre conformément à ce qui est juste, qu'aux ajouts mythologiques faits à une narration qui ne perd pas de vue (si elle ne se fonde pas sur) ce que font les hommes. La première remarque sur le début de la description du Tartare consiste à dire qu'elle « convient bien pour des tortures et des supplices » (I.V. I, p. 581, 1. 15-16).

34. L'expression poenae propositae ex cotidiana meditatione m'a menée à cette hypothèse. Il est vrai que, dans le contexte étroit (avec la référence, un peu plus bas, aux actus hominum , qui s'opposent aux actus inferos auxquels Virgile ne s'est pas vraiment intéressé, selon $\mathrm{TCD}$, mais auxquels il a tout de même fait une part, en poète - ex mendacio poetae), meditatio cotidiana semble devoir davantage référer à une pratique (ce que les hommes pouvaient voir, en termes de supplices, tous les jours) qu'à un exercice de méditation quotidienne. JEANJEAN 2010 traduit ainsi : « les châtiments représentés par ce que l'on voit tous les jours ». Un tel emploi de meditatio (où il ne s'agit plus d'une pratique, dans le sens d'un exercice, mais celui d'un usage) ne se retrouve cependant pas ailleurs dans les Interpretationes Vergilianae. En outre, dans un contexte élargi, et si l'on pense aux interprétations néo-platoniciennes et aux appropriations chrétiennes qui ont pu être faites du chant 6 de l'Énéide, l'expression fait songer à l'exercice spirituel consistant à s'infliger la vue - représentation mentale et/ou, parfois, vision de figurations littéraires ou artistiques - des crimes et des châtiments de manière purgative. Voir GOSSEREZ 2001 : p. 97-98. Parmi les passages où l'on retrouve l'expression proponere poenas, il en est un qu'il semble particulièrement intéressant de confronter au nôtre: il se trouve dans la traduction des Recognitiones pseudo-clémentines par Rufin (Rec. IX, 11, 4: Die Pseudoklementinen, II, Rekognitionen in Rufins übeersetzung, édition de B. Rehm, revue par G. Strecker, Berlin, Akademie Verlag, 1994), et consiste à affirmer le pouvoir de la pénitence et l'efficacité de la peur que l'on ressent en se mettant devant les yeux les supplices des hommes, bien plus que si l'on voyait le feu de l'enfer et le « Pyriflegethon » - qui est exclu de la discussion (Denique nunc nolo Pyrifleget<h>onta adducas in medium; hominum tibi propone poenas, et uide quantum ualeat metus; potest ne quisquam, cum pro amoris 
crimine ad supplicium ducitur et ad stipitem conligatur urendus, in illo tempore eius quam amauit uel desiderium capere uel speciem ante oculos ponere?) En défendant l'utilité du poetae mendacium, TCD réintroduit en quelque sorte le Pyriphlégéthon dans le processus.

35. Ce discours d'Anchise sur le futur de Rome est d'ailleurs présenté comme une spécification de la prophétie de Jupiter au chant 1 (I.V. I, p. 605, 1.25-27, où l'on retrouve le couple specialiter / generaliter) : ici il va être question de personnes, alors que là il s'agissait surtout de tempora regnandi.

36. Voir ainsi I.V. I, p. 550, 1.5-11, p. 563, 1. 11-15: le commentateur y explicite la miseratio avec laquelle Virgile évoque certains morts (les âmes qui se pressent pour être transportées par Charon, les nouveau-nés).

37. Voir d'abord la Vie de Virgile par TCD, 32.

38. Vita Donatiana, 11.

39. C'est une interprétation à laquelle se prête particulièrement la version donnée de la scène par le chevalier Wicar, telle qu'on peut la voir au Palais des Beaux-arts de Lille (J.-B. Wicar, Virgile lisant l'Énéide devant Auguste, Livie et Octavie, premier quart du XIX siècle, inv. P. 442). Je dois à Ioannis Ziogas de m'avoir fait reconsidérer cet épisode remarquable de la tradition biographique virgilienne grâce à la présentation qu'il lui a consacrée lors du Symposium Cumanum de 2013 (voir note 1 supra).

40. I.V. I, p. 617, 1. 26-p. 618, 1. 2.

41. TCD insiste sur le sens adverbial de recto dans recto litore au v. 900, pour dire le trajet direct qu'Énée fait jusqu'au port de Gaète : il le fait à juste titre et à l'image de la narration idéale du chant 6 , spécialement marquée par la hâte.

42. C'est le treizième livre qu'il annonce à la fin de son commentaire aux v. 641-646 du chant 7 de l'Énéide, pour un travail complémentaire qu'il présente aussi dans une lettre adressée à son fils à la fin de son œuvre. Sur ces déclarations, voir PIROVANo 2010.

43. En souvenir du titre de DEREMETZ 1993 et du rapport vraisemblablement instauré par le poème virgilien entre le labyrinthe de Crète et le cheminement aux enfers.

\section{RÉSUMÉS}

Dans son commentaire de l'Énéide, Tiberius Claudius Donat ne considère pas qu'il y a un sens caché à dévoiler dans le texte de Virgile. Dans la perspective rhétorique qui lui est propre, et au moyen du travail de reformulation, ce commentateur exprime au sens étymologique, "fait sortir » des choses que Virgile a formulées de manière admirablement condensée dans son texte ; il lui arrive ainsi souvent de s'éloigner de la lettre de ce texte, mais ce n'est pas dans une démarche explicitement allégorique ou dans l'idée que Virgile a ainsi masqué, par exemple, une philosophie. Dans ces conditions, on peut déjà se demander ce que ce commentateur a fait du chant 6 de l'Énéide. Et la question se pose de manière d'autant plus vive qu'à l'occasion, au nom de la cohérence du texte, Tiberius Claudius Donat semble prêt à mettre le passage sur les enfers entre parenthèses, à en faire abstraction. Dans le détail du commentaire du chant 6 , cette indifférence ne se vérifie pas; l'on y trouve plusieurs analyses intéressantes (sur le rythme et le ton du récit notamment), même si, sur le fond, la nature propre de la catabase contraint Donat à rester encore plus qu'il ne le voudrait, sans doute, à la surface du texte. 
Unlike many ancient commentators, Tiberius Claudius Donatus in his Virgilian commentary (the Interpretationes Vergilianae) does not aim at unveiling some of the religious or philosophical facts or truths that Virgil is meant to have deposited into his text. In his paraphrasis he does work the words of Virgil in order to make them express all that they contain (many things, given Virgil's mastery in regard to breuitas)... and often more. But Donatus is not a true allegorist, and he usually leaves out mythological as well as historical information. In these circumstances one may wonder about the way he has addressed the sixth book of the Aeneid and the episode of Aeneas' descent into the Underworld in particular. This question arises more powerfully at the beginning of the seventh book, when Donatus seems ready to turn the catabasis into a superfluous digression. This view is not found as it is in the main commentary of Book 6 , where the commentator is faithful to his rhetorical and moralistic program of reading and proposes many interesting remarks. Because of this program, he may have experienced some difficulty in front of the exceptional richness of this book, which he looks very conscious of.

\section{INDEX}

Mots-clés : Tiberius Claudius Donat, commentaires virgiliens, catabase, digression, rhétorique

Keywords : Tiberius Claudius Donatus, Virgilian Commentaries, catabasis, Digression, rhetoric

\section{AUTEUR}

\section{SÉVERINE CLÉMENT-TARANTINO}

MCF en langue et littérature latines à l'Université de Lille (Lille 3, UFR Humanités-Équipe HALMA, UMR 8164). Virgilienne et passionnée par l'épopée gréco-latine au départ, elle travaille aussi beaucoup depuis dix ans sur la réception de Virgile et la tradition des commentaires virgiliens (voir par exemple la traduction du commentaire de Servius au livre 1 de l'Énéide, accomplie avec Alban Baudou et parue sous le titre : À l'école de Virgile, Presses du Septentrion, 2015, et l'ouvrage collectif La représentation du " couple »Virgile-Ovide dans la tradition culturelle de l'Antiquité à nos jours , Presses du Septentrion, 2015, édité en collaboration avec Florence Klein). 